\title{
ARNAU DE VILANOVA. LA MEDICINA, LA CIENCIA Y LA TÉCNICA EN TIEMPOS DE JAIME II
}

\author{
Emili BALAGUER I PERIGÜELL \\ Catedrático de Historia de la Ciencia
}

Cuando a mediados del siglo XIII, Jaume I conquista el territorio que se configura como Reino de Valencia en el seno de la Corona de Aragón, comienza a iniciarse el segundo de los períodos que suele distinguirse en la actividad científica, desarrollada en Europa occidental, a lo largo de la edad media: el proceso de asimilación, a través de los textos árabes, del saber clásico griego y helenístico enriquecidas en el mundo islámico por aportaciones originales y elementos de la ciencia clásica india.

Este proceso que se inició principalmente con las traducciones de textos astronómicos y matemáticos realizados en los monasterios catalanes de Vic y Ripoll, cuyos abades mantuvieron contacto con la Córdoba del califato, mediante la ayuda de los mozárabes; y en el campo médico por las realizadas en la ciudad italiana de Salerno, primera escuela médica laica de la Europa medieval. Pero desde mediados del siglo XII, fue indiscutiblemente Toledo la capital fundamental de la transmisión al occidente europeo del saber greco-árabe.

Pero además durante los siglos XI y XII, la Península Ibérica se convirtió en el escenario más importante de la cultura islámica, tanto en el campo de las matemáticas, la astronomía, la geografía, etc. como en el de la botánica, la agronomía y la medicina (Averroes y Avenzoar). Las aportaciones fueron tan importantes que este período andalusí constituye una etapa con personalidad propia en la historia de la ciencia.

El actual territorio valenciano permaneció al margen de este proceso, ocupado por grupos berberiscos en los que apenas debieron tener vigencia los elementos árabes, destruyeron la organización sociopolítica visigoda, implantando una sociedad primitiva casi exclusivamente rural. Sólo a partir del siglo XI, con la constitución de los reinos de Taifas, fundamentalmente los de Valencia y Denia, se detecta una recuperación de la actividad científica. 
El núcleo de mayor importancia fue Denia, sobre todo a partir del siglo XI en el reinado de Muŷāhid. En la corte de este monarca, que poseía una sólida preparación cultural, se reunieron científicos e intelectuales procedentes de diferentes lugares de al-Andalus. Entre ellos cabe destacar al astrónomo Ahmad Ibn al Saffār, autor de un tratado sobre el astrolabio, traducido al catalán por Millàs Vallicrosa, y que tuvo gran difusión tanto en el mundo árabe como en el latino.

Después de haberse formado en el Oriente islámico, se instaló en Denia el médico Abū Marwan ibn Zuhr, que alcanzó gran prestigio como clínico, Su hijo Abū-'Alā'zuh (el Albuleizar de los latinos), es autor de diversas obras de las cuales una monografía sobre la litiasis renal y otra sobre la higiene individual, sólo se conserva el texto latino. La obra más destacada, editada en árabe y francés en 1911 por G. Colin, es la titulada Tadhkira, compendio de medicina práctica con una orientación puramente galénica y ambientalista en la mejor tradición hipocrática. La dedica a su hijo, Abū Mazwān ibn Zuhr (el Avenzoar latino), que a su vez es autor de la más importante contribución andalusí a la medicina clínica, sólo comparable a la de Rhazes.

Mención especial merece Abū-s-Salt Umayya, nacido y residente en Denia hasta los veinte años. Se trata del tipo de médico con una amplia preparación intelectual, científica y técnica, característica del mundo árabe medieval. La numerosa producción escrita de Abu-s-Salt comprende una tratado de geometría, otro de astronomía y una monografía sobre el ecuatorio, un instrumento de invención andalusí para calcular la posición de los planetas. Su principal obra médica es un tratado de medicamentos simples, estudiado en 1883 por M. Steinschneider partiendo de un ejemplar manuscrito, al que recientemente se ha sumado otro descubierto por F. Giron y L. García Ballesta. Se le considera como un hito importante en la trayectoria de la farmacoterapia al al-Andalus y fue traducido al hebreo por Jehuda Ben Salomon Natan y al latín por Arnau de Vilanova. Es autor, también, de un estudio sobre geografía física y la cultura de Egipto, de un tratado de lógica, de una epístola sobre la música y de una antología de poemas de autores andalusís.

En el reino de Taifas de Valencia, las figuras más tempranas dignas de mención fueron el constructor de instrumentos astronómicos Ibn Sā id al-Shali, que desarrolló su actividad en la segunda mitad del siglo XI, autor de uno de los primeros globos celestes que se conservan. Fue construido en Valencia en el 1102 y actualmente se encuentra en el Museo de Historia de la Ciencia en Florencia. Junto al-Shalí, hemos de mencionar al matemático lbn Sayyid al-Kallū, que desde los años centrales del siglo XI hasta su muerte, que J. Vernet sitúa hacia 1119 en Játiva, contribuyó a la teoría pitagorística de los números procedente de Nicómaco de Gerasa, matemático griego del siglo II y que destacó muy especialmente como geómetra, sobre todo en relación con las Crónicas de Apolonio de Pérgamo, el gran título helenístico de la disciplina.

El principal discípulo de al-Kalbū fue el zaragozano Ibn Baŷya (el Avempace latino) autor de varios tratados científicos y ampliamente conocido por sus obras filosóficas, en especial la traducida al castellano con el título de Régimen del solitario, que tuvo una gran influencia en el pensamiento europeo no sólo en la edad media sino, incluso, en los primeros tiempos modernos. Después de un largo peregrinaje por la Península 
Ibérica y el norte de África, Avempace residió, durante la segunda década del siglo XII en Valencia y Játiva, donde fue acusado de hereje.

Coetáneo de al-Kalbū, fue el médico valenciano 'Abd-al Wadūd b Abd-al Malik, autor de una obra en defensa de la medicina científica, contra los teólogos que la consideraban un desafío de la omnipotencia divina y contra las prácticas empíricas sin fundamento racional. García Ballester ha destacado el interés de este texto para conocer los problemas sociales, económicos y éticos del ejercicio médico en el mundo islámico.

En el ocaso del dominio musulmán en territorios valencianos, J. Vernet ha estudiado un grupo de científicos, fundamentalmente matemáticos, que desde finales del-siglo XI, hasta la primera mitad del siglo XIII, suponen una verdadera avanzada, en dicha área, en el panorama general andalusí. La conquista cristiana obligó a muchos de ellos a emigrar a Granada, Sevilla u otras localidades meridionales. Algunos murieron poco antes de ver destruido su mundo sociopolítico y cultural. Es el caso del médico alcireño ibn Tumlus, autor de un libro parcialmente editado y traducido por $M$. Asin Palacios, con el título de Introducción al arte de la lógica, que murió en su ciudad natal en 1223; y del matemático saguntino al-Quda ci, muerto en Valencia en 1230.

La conquista por Jaime I significó un cambio drástico en la sociedad valenciana de mediados del siglo XIII. La entrada de una nueva cultura - la cristiana - en el seno de la cultura islámica, hasta ese momento hegemónica, conllevó la presencia de elementos nuevos no sólo en los contenidos científicos sino también en el modo de concebirlos. Pero no olvidemos que tanto en la sociedad islámica - que va a ser profundamente alterada- como en la nueva sociedad cristiana que se inicia en 1238, existió la cultura judía. A partir de los años de la conquista, la sociedad valenciana medieval sólo podrá entenderse teniendo en cuenta este entramado complejo de tres culturas.

A pesar del trauma de la conquista, la comunidad mudéjar valenciana conservó estructuras que permitieron un cierto nivel cultural. Es el caso de las «aljamas» independientes como lugares privilegiados donde las leyes y costumbres islámicas continuaban vigentes, asesoradas por una asamblea de notables. En estos momentos no debe confundir «las aljamas» con lo que más adelante serás las «morerías», verdaderos ghettos para mantener aislada a la comunidad musulmana. Mikel de Epalza interpreta esas asambleas como el grupo de notables que entregaron cada una de las plazas, por lo cual fueron elevados por los cristianos a un cuerpo parecido al de los consejo municipales. Por otra parte las ansias evangelizadoras de las primeras jerarquías eclesiásticas, tampoco parece ser tuvieron éxito y buena prueba de ello, es la desaparición en 1314 de las escuelas de árabe y hebreo, fundadas en Játiva veinticinco años antes para la formación de misiones. Sin embargo, los musulmanes valencianos no tuvieron una «madrasa» o centro de estudios islámicos, como la tuvieron los de Zaragoza, con influencia en todo el Reino de Aragón, donde se enseñó medicina hasta finales del siglo XV.

La degradación de la cultura musulmana en el Reino de Valencia, no fue tan rápida como se creía y a pesar de las prohibiciones legales, como la de 1388, que no permitía a los médicos musulmanes asistir a enfermos cristianos, sus servicios fueron soli- 
citados por municipios, hospitales, nobles y reyes. Por ejemplo, Juan I solicitó en 1387 desde Barcelona, la presencia del médico valenciano Abrahim y el Hospital de la Reina de la ciudad de Valencia, encargó a un «metge moro l'assistència a un pobre malalt, i va destacar que va ser més eficaç que el metge cristià assalariat de manera regular» (J.M. López Piñero y V. Navarro).

Un segundo aspecto de la actividad científica de los mudéjares valencianos, fue la desarrollada por los alfaquíes que mantuvieron relaciones con la cultura del mundo islámico del norte de África y del Oriente. Como ejemplo notable nos referiremos al alfaquí de Paterna, que en los años centrales del siglo $\mathrm{XV}$, aprovechando un viaje a $\mathrm{El}$ Cairo —el importante centro de ciencia islámica del Mediterráneo oriental— se trajo consigo un manuscrito científico innovador en el campo de la trigonometría y que permitía mediante un instrumento utilizado por los astrónomos de El Cairo, conocido como sexagenarium y mediante la aplicación gráfica de fórmulas trigonométricas, dentro de la astronomía ptolemaica, calculaba las ecuaciones del centro de la tierra y el argumento de los planetas. Fue traducido al catalán en Paterna en 1456 y al latín por el médico valenciano Joan de Bosnia, uno de los miembros fundadores de la Escuela de Cirugía Valenciana en 1462.

Sin embargo, es preciso matizar los comportamientos en la actividad científica ya que de hecho las tres culturas presentes en tierras valencianas no se comportan como departamentos estancos. Si en el mundo cristiano despertó gran interés, incluso en fechas tardías como ya hemos visto, algunos aspectos de la ciencia árabe; la corriente contraria también tuvo lugar. La asimilación de la ciencia greco-árabe por la cultura latina y su estructuración entorno a planteamientos cristianos, el llamado movimiento escolástico; tuvo su influencia en la cultura árabe. Un ejemplo paradigmático es la trayectoria del médico Muhammad al-Šafra, nacido a finales del siglo XIII en la pequeña señoría musulmana de Crevillente y residió en ella hasta su desaparición en 1318. Fue autor de un tratado de cirugía basado principalmente en la obra de Albucasis, cuyo contenido amplió en diversos temas, y en la misma línea que éste denunció las prácticas empíricas sin preparación cultural. La relación de al-Saafra con la cultura científica cristiana consistió no sólo en tener un maestro cristiano, un tal Bernard, sino en que su obra responde a lo que García Ballester llama el «reflujo de la escolástica» ya que algunos autores musulmanes, como el médico de Crevillente, asimilaban los planteamientos cristianos bajomedievales.

Los judíos constituyeron en la baja edad media valenciana una minoría que aumentó su importancia a lo largo de siglo y medio que siguió a la conquista, a causa de la mayor permisividad cristiana en este período, en comparación a la intolerancia almohade anterior. En el Llibre del Repartiment figuran tres alfaquíes judíos, médicos, vinculados a la casa real. Aunque en el sínodo de la iglesia valenciana de 1263 se acordó "que cap clergue ni laic demane en les seues malalties un metge jueu o en reba medicació", algunos de los alfaquíes judíos ejercieron complejas funciones en el seno del círculo real. Samuel Abenmenassé, fue nombrado por Pedro III en 1279, médico real e intérprete oficial del árabe. Poseyó estos cargos hasta 1286 en que pasa a ejercerlo en la corte del infante Alfonso, hasta que le sucedió en estas funciones el 
judío catalán Baoudavid Bonsenyor. Jahudá Bonsenyor, hermano de éste, realizó los mismos servicios en la corte de Jaime II. El cargo de intérprete de árabe no se limitaba a funciones puramente administrativas o diplomáticas, sino que se implicaba también en el campo científico, como lo demuestra la traducción que Jahudá hizo para Jaime II, del árabe al catalán, de la obra de Albucasis y como estuvo de atento este monarca, mediante el alfaquí, a la literatura médica árabe existente en sus reinos, de la cual intentó dotar al reciente Estudio General de Lérida.

La actividad de Samuel Abenmenassé en la corte, justo los mismos años que Arnau de Vilanova vivía en Valencia y Barcelona - también en el círculo cortesano de esta ciudad-sugiere una posible relación entre los dos. Mucho más si se considera que fue durante estos años que Arnau tradujo del árabe al latín diversos escritos médicos, entre ellos el tratado sobre medicamentos simples del médico valenciano Abu Salt, ya citado anteriormente.

La situación de la minoría judía empeoró drásticamente a finales del siglo XIV, especialmente a partir de los asaltos de la judería y las matanzas de 1391. La progresiva intolerancia desde estos acontecimientos obligó a mucha gente a exilarse, otros se convirtieron al cristianismo aunque sólo fuera por guardar las apariencias y con la exclusiva finalidad de poder integrarse en la sociedad cristiana, lo que podemos ejemplificar en el médico setabense Lluis Alcanyis, primer catedrático de medicina de la Universidad de Valencia y autor de un Regiment preservatiu i curatiu de la pestilència, cuyo trágico final fue ser condenado a la hoguera.

Mientras que en la Europa occidental cristiana, el proceso de transmisión de la ciencia greco-árabe, culmina, en parte, en la creación de una nueva institución para su cultivo y transmisión, el Studium generale o universidad; el modelo de enseñanza implantado en el Reino de Valencia, por los Furs de Jaime I debía ajustarse al conocido como «modelo abierto» de origen islámico: «Tot clergue o altre hom pusque franchament e sens tot servei e tribut tenir estudi de gramatica e de totes altres arts e de fisica (medicina) e de dret civil e canonich en tot loch per tota la ciutat». No obstante, la iglesia mostró muy pronto su interés por controlar la cultura. Con esta intención se fundó en 1254 en la catedral de Valencia una escuela de gramática, además de conseguir nueve años más tarde un privilegio pontificio para fundar un Studium generale, que quedó en mero proyecto. La iniciativa partió de Andreu Albalat, que ocupó la sede episcopal valenciana desde 1248 hasta 1276 y que García Ballester considera posible que interviniera en favor del mencionado privilegio, ya que el 1245 vivía en Roma. Albalat participaba de la mentalidad de los dominicos de la época, que había encabezado san Alberto Magno, favorable a la ciencia desde los supuestos aristotélicos. Durante su estancia en Roma hizo amistad con Teodorico Borgognoni, también dominico y autor del primer tratado de cirugía importante aparecido en el ambiente de la Universidad de Bolonia, basado directamente en la obra de Albucasis. La versión definitiva de esa cirugía fue redactada a instancias de Andreu Albalat y dedicada por su autor al obispo de Valencia.

El sucesor de Albalat en la sede episcopal valenciana, Jaspert de Botonac, mantuvo también una postura favorable a la ciencia, lo que justifica el hecho de que Arnau 
de Vilanova le dedicara la obra De improbatione maleficorum. Sin embargo en el siglo siguiente se detecta, entre algunos representantes del mundo eclesiástico valenciano, una actitud de reserva hacia la autonomía del pensamiento racional y un mayor énfasis en la necesaria subordinación del pensamiento científico a la teología. Debió influir en esta actitud las condenas por el obispo de París, Etienn Tempier, de una larga lista de postulados aristotélicos considerados contrarios a la fe cristiana; así como la vinculación de ciertas materias como la alquimia y la astrología a la tradición cultural árabe. Los principales representantes en Valencia de esa actitud fueron el franciscano Francesc Eiximenis y los dominicos san Vicent Ferrer y Antoni Canals. Fueron hombres prácticos que supieron construir, mediante la predicación y su comprometida implicación en la vida más real e íntima de la ciudad de Valencia, un puente entre las ideas teológicas conservadoras y el pueblo, tanto el patriciado como en el resto de la población. Es en este contexto en el que hay que situar los consejos de Eiximenis a los hijos del patriciado urbano valenciano, previniéndoles contra el estudio de «artes liberales» como la aritmética, la geometría, la astronomía, etc., por no ser ciencias religiosas y conllevar el peligro de perder la fe y pasarse a «serraïns»; san Vicent Ferrer, prior del influyente convento dominico de Valencia, no dudó en condenar a Aristóteles y Plantón al infierno; e igualmente el dominico Antoni Canals, que insistió en la inestabilidad de una fe basada en los estudios y la lectura de libros. Dentro de este ambiente, no podemos olvidar las recomendaciones del obispo de Valencia, en 1399, al abad del monasterio de San Jerónimo de Gandía cuando le puso en guardia contra la "ciencia luliana» y le aconsejó "no recurrir a los laberintos de la ciencia, sino a la instrucción moral...».

Investigaciones sobre el siglo XIV valenciano, han puesto de manifiesto la existencia en la ciudad de Valencia, a partir ya de los años finales del siglo XIII, de un grupo social muy vigoroso que dotó de un fuerte dinamismo a la vida social y económica valenciana. Un grupo social que, como en otras ciudades europeas, se hizo con el gobierno de la ciudad y que estuvo formado por artesanos, mercaderes, profesionales y rentistas no nobles: los civi Valentie o "ciutadans de València» de los documentos bajomedievales. Ese grupo social estuvo especialmente preocupado por los problemas médicos e incluso, tomó partido muy pronto (1329) por el modelo escolástico de profesional médico salido de aulas universitarias. Fue también el grupo social responsable de cierto grado de medicalización que tuvo lugar en la Valencia bajomedieval, es decir, de la paulatina conversión de la salud en un problema técnicamente médico. A ellos se debió la introducción de una serie de medidas, algunas de cierta audacia tecnológica, que fueron más allá del individuo y de los estrechos círculos cortesanos o adinerados: control de alimentos, medidas para evitar la contaminación del agua de uso doméstico, así como para procurar la evacuación de aguas sucias y limpieza de basuras, desecación de marjales, control de los profesionales sanitarios, introducción de profesionales médicos en los hospitales, etc.

Desde su monopolio del poder municipal, esta oligarquía se posicionó a favor de la institucionalización de la enseñanza, la cultura y la ciencia, como ya hemos indicado anteriormente, de acuerdo con el modelo universitario. Los nuevos fueros de Alfonso I (1329) consagraron la sanción normativa de este modelo; a pesar de ello, el nuevo 
proyecto de fundar un studium generale (1373), se frustró una fez más. La pugna entre la iglesia y la oligarquía urbana en torno al control de la enseñanza, fue, sin duda, uno de los factores determinantes que provocaron que Valencia no tuviera un Studium generale durante la baja edad media. Frente a las escuelas catedralicia y conventuales, el municipio tenía "escoles de gramatica e logica e de altres arts", que van a ser dotadas de un local propio en 1374 y unificadas, sin tomar en consideración la opinión del obispo, en 1411. Un acontecimiento de mayor importancia fue la creación en 1462 de la escuela de cirugía, de la que hablaremos luego, el desarrollo de la misma corresponde ya al período protorrenacentista.

Al complejo panorama bajomedieval que acabamos de describir, hay que añadir la enseñanza en Valencia de las doctrinas de Ramón Lull. Un primer lulismo entroncará muy pronto con la espiritualidad arnaldiana y a pesar de los esfuerzos del inquisidor de la Corona de Aragón Nicolau Eimeric y la ofensiva de los religiosos opuestos al cultivo de la ciencia; no conseguirán impedir que en 1369, Pedro III, concediera un privilegio a Berenguer Fluviá para enseñarlas en el campo de la filosofía, la astronomía, la medicina y dar el título de maestros en estas materias. Como han estudiado L. Comenge y T. y J. Carreras Artau el centro principal del poderoso movimiento lulista fue la ciudad de Valencia y la primera institución docente lulista fue la escuela dirigida en Alcoy por Pere Rosell, amparándose en el privilegio de Pedro III.

La producción científica de los cristianos valencianos a lo largo de la baja edad media, se llevó a cabo, como es lógico, paralelamente a la trayectoria de los condicionamientos socioculturales y políticos que sumariamente acabamos de recordar. Comenzó siendo la propia de una zona de frontera o regne de croada, como le gusta decir a R.I. Burns, la de unos territorios conquistados por las armas dominando la población autóctona que progresivamente se irá degradando hasta su expulsión definitiva. Los descendientes de los conquistadores irán progresivamente integrando la actividad científica de los cristianos valencianos en el mundo europeo occidental, no sólo en cuanto a su organización, sino también en lo relacionado con el producto. Ahora bien, conviene recordar que esta integración no fue completa hasta el período renacentista.

Desde esta perspectiva podemos distinguir dos etapas en la producción científica de los cristianos valencianos a lo largo de la baja edad media. La primera abarca desde la conquista a finales del siglo XIV y se caracteriza por una estrecha vinculación con la universidad de Montpellier, perteneciente a la Corona de Aragón, quedando en un segundo plano la universidad de Lérida, fundada por Jaime II (1300). En este período la convivencia con los musulmanes y judíos todavía tiene un peso importante. La segunda etapa comprende desde finales del siglo XIV, coincidiendo con la desarticulación de la comunidad judía y de que Montpellier deja de pertenecer a la Corona de Aragón, hasta las décadas centrales del siglo XV. En este período, aunque no se rompe definitivamente las relaciones con Montpellier, pasaron a primer término como modelo de organización para la oligarquía urbana local, las universidades de Bolonia, Pisa, Siena y Padua.

La primera etapa se inició con la producción de Arnau de Vilanova, la figura más importante con gran diferencia de todo el período medieval. 
Arnau fue miembro de una familia catalana, hijo quizá de aquellos primeros repobladores del recién conquistado Reino de Valencia, donde parece que nació entre 1238 y 1240 . Si discutible es el lugar de su nacimiento, no cabe la menor duda sobre su vinculación a Valencia, ciudad en la que poseía importantes bienes, amigos y seguidores; a la que volvió repetidamente a lo largo de su vida y donde fue asignado por Clemente $V$ al nombrarle clérigo de la diócesis valentina.

Según su propio testimonio, sabemos que comenzó su formación con los predicadores, estudió medicina en Montpellier hacia los años sesenta y allí se casó con Agnés de Blasi, hija de un famoso comerciante, J.A. Paniagua, uno de los principales especialistas en este autor, no cree que ampliara su formación en Nápoles, como algunos han sostenido, sino que ejercería como médico en Valencia hasta su marcha a BarceIona, en 1281, como médico de cámara del rey Pedro III.

Hacia 1289 inició su colaboración con la universidad de Montpellier, si bien no fue hasta 1295 cuando manifestó un claro interés por instalarse allí, constituyendo un patrimonio y, paralelamente abandonó la gestión de sus negocios en Valencia en manos de procuradores. En 1309 abandonó la Universidad, el mismo año que su amigo, el papa Clemente $\mathrm{V}$ promulgó una bula que ordenaba el plan de estudios médicos en Montpellier y en cuya gestación fue fundamental la influencia de Arnau, médico suyo en aquella época.

Una desgraciada intervención ante la Curia de Avignon en verano de 1309, donde expuso sus conocidas y controvertidas opiniones religiosas, le supuso el ostracismo y la animadversión del su principal valedor y protector, el rey Jaime II. Arnau se instaló junto a Federico III, rey de Sicilia y el más ilustre seguidor de las propuestas espirituales de este autor, hasta su muerte, ocurrida frente a Génova el 6 de septiembre de 1311, cuando se dirigía a Avignon.

J.A. Paniagua ha puesto de relieve la trascendencia de la personalidad de Arnau: «Ningún otro comparable a él se encuentra en el conjunto de los reinos de la Reconquista, y muy pocos se le asemejan en la España musulmana y en la Cristiandad medieval. La figura de Mestre Arnau de Vilanova destaca claramente como una de las más notables y representativas de la ciencia médica en el ámbito entero de la Edad Media universal».

Arnau aparece como un hombre de frontera, que manifestó una clara vinculación al arabismo y al escolasticismo. El saber escolástico estaba fundamentado en el criterio de autoridad, según el cual, las afirmaciones de los clásicos no necesitaban ser contrastadas con la realidad. En tal ambiente, el descubrimiento de la ciencia árabe comportó una auténtica revolución mental, ya que se constituyó como nueva autoridad, planteando al hombre medieval la necesidad de conciliar entre las contradicciones existentes en las opiniones de autoridades igualmente respetables mediante una hábil utilización de la ratio.

Así fue acuñando el método de las questiones disputata para la enseñanza, según el cual se discutía sobre una base textual, utilizando la lógica deductiva.

La labor científica de Arnau fue desarroliada fuera de Valencia y se caracterizó por su vinculación total al movimiento escolástico. Por un lado, contribuyó a ampliar el 
conocimiento clásico mediante la traducción del Liber de viribus cordis de Avicena y el Liber de rigore et tremore et iectigatione et spasmo de Galeno. Por otro, fue uno de los principales introductores del llamado "nuevo Galeno" en las facultades de medicina europeas, tal y como ha puesto de relieve L. García Baliester.

Su nombramiento como médico de cámara iba a ofrecer la posibilidad de iniciar su actividad científica, pues Pedro III marchó al año siguiente (1282) a Sicilia para ser coronado rey. Buen conocedor del árabe, aprovechó las posibilidades que le ofrecían la rica biblioteca de la corte y, posiblemente, la presencia de Samuel Abenmenassé. La inmensa tarea llevada a cabo por las escuelas de traductores, dio lugar a que el trabajo de Arnau hubiera de reducirse a textos secundarios, aunque de indudable valor, que completaron el proceso de recuperación del saber greco-árabe.

Ahora bien, los escritos que le dieron auténtica fama, fueron sus obras originales y versiones de textos hipocráticos y galénicos elaboradas, en su mayor parte, en Montpellier durante su etapa universitaria.

A mediados del siglo XII, parece que el Corpus Toletanum ya estaba plenamente asimilado en Montpellier, con lo que se añadía la filosofía natural de Aristóteles y el Canon de Avicena a la formación del médico.

A finales del siglo XIII, es decir, en la época en que Arnau enseñaba, confluyeron en Montpellier dos tradiciones renovadoras: el arabismo, de honda raíz montepelusana, y el escolasticismo médico italiano de la bolonia de Taddeo Alderotti y en la que el Canon era fuertemente cuestionado como texto central para la enseñanza. Así se cerró el proceso de asimilación plena del galenismo iniciado con la Escuela de Traductores de Toledo, con lo que se perfeccionaron enormemente los conocimientos sobre la fisiología, patología, clínica y terapéutica galénicas entre los profesores universitarios. L. García Ballester centra el protagonismo de Arnau en cuatro puntos: su labor universitaria de comentarista de los tratados de Galeno; su aportación a la empresa de transmisión, en la que fue fundamental su conocimiento directo de las fuentes árabes; su intervención en las ordenaciones que regularon los estudios médicos de Montepellier desde 1309, y su indiferencia o conformismo ante la obra morfológica de Galeno.

Parte de sus escritos respondían a un esquema metodológico basado en la total confianza en la vía experimental, al permitir conocer lo sensible en su manifestación concreta, frente a la razón que sólo nos informa de las propiedades comunes. Tal confianza debía ir acompañada de una cautela frente a los resultados, pues el proceso experimental está sujeto a multitud de factores que pueden perturbarlo. Por último, una prudente actitud ante la autoridad científica, que le empujó a condenar los excesos de la especulación filosófica —en especial de los averroístas- y las síntesis médicas demasiado elaboradas —el Canon principalmente- pues coartaban la necesaria curiosidad de acudir a los textos originales. Por ello, algunas de sus obras manifiestan un claro sentido polémico.

Tal actitud se manifestó también en sus escritos de práctica terapéutica, donde dictaba sensatas dietas, recomendaba medicamentos de propiedades suficientemente comprobadas y se mostraba contrario a los abusos de las sangrías.

Arnau defendía que Hipócrates y Galeno habían transmitido la técnica y el método 
idóneos para el mantenimiento y restablecimiento de la salud, objetivo prioritario de la medicina. Por ello, desplegó una interesante actividad como comentarista, que en el caso de Hipócrates se limitó a la Articella. Ante la evidente insuficiencia de estos textos para cumplir su propósito, dedicó una gran atención a las obras patológicas y clínicas de Galeno, adelantándose a los esfuerzos boloñeses en varias décadas. Podemos destacar su versión De inequali intemperie, breve tratado galénico, al que hizo un comentario extenso y complejo, abordando el concepto de complexión patológica -fundamental en la patología humoralista- pues lo concibió como una introducción a los dos grandes textos terapéuticos de Galeno: De simplicibus medicinis y De ingenio sanitatis, este último también comentado por él.

Llama la atención el empeño que Arnau pareció mostrar en evitar al máximo la difusión de estos escritos, con el fin de ocultar información a sus enemigos académicos, los averroistas, quienes constituían un importante grupo de presión universitario.

Entre sus trabajos originales destacan el Speculum medicinae. Obra de madurez, aparece como uno de sus libros más extensos e importantes, donde se refleja claramente la doctrina médica de su autor, Se trata de un texto de medicina básica donde ofrece un resumen de fisiología, higiene fundamental, farmacología general y psicopatología básica. Su intención es puramente didáctica, exponiendo y completando de forma sencilla y clara las propuestas galénicas.

Este texto se complementa con las Medicationis parabolae, las cuales tuvieron una inmensa difusión. De forma aforística, estudia los modos de enfermar generales, las enfermedades simples y algunos consejos terapéuticos.

La farmacología fue la disciplina que posiblemente mayor prestigio concedió al médico valenciano. Hallamos escritos de base teórica, como el ortodoxamente galénico De simplicibus; y de contenido eminentemente práctico, como el De gradibus. En este famoso tratado propuso la aplicación del cálculo matemático para determinar la dosificación de los medicamentos compuestos, siguiendo los métodos del Al-Kindi.

La obra de mayor difusión fue el Antidotarium auténtica enciclopedia que incluye varios capítulos de farmacología general, seguidos de un importante apartado práctico, donde se indica la definición, composición y procedimiento de confección de multitud de electuarios, píldoras, jarabes, emplastos, etc. Esta colección de fórmulas parece responder a una reelaboración del Antidotarium Nicolai, quizá el escrito original más famoso salido de la escuela de Salerno.

Por último, el Regimen sanitatis fue el texto de Arnau más conocido. En efecto, el médico valenciano se convirtió en uno de los clásicos de la literatura higiénico-dietética medieval. Este género alcanzó una gran popularidad por la escasez de remedios específicos de tratamiento con que contaba la medicina de aquel período.

Llama la atención que un tratado de higiene individual confeccionado expresamente para la persona del rey Jaime II, alcanzase tan gran reputación y difusión. Trata su autor aspectos tales como las características idóneas del lugar donde instalar la corte, de los cuidados personales - ejercicios físicos, aseo, estados de vigilia y sueño, emociones - y régimen alimenticio — cualidades salutíferas de los principales alimentos y bebidas-. El libro está escrito en un lenguaje sencillo y escueto, despojado de 
toda erudición, manifestando la inmensa capacidad de Arnau para hacerle comprender por los profanos. Esta claridad de estilo, unido a la utilidad de su doctrina, explican que fuera uno de los textos más leídos de la Edad Media.

Arnau aparece, pues, como uno de los médicos más famosos, tanto desde su faceta de médico práctico, como en la académica. Sus obras fueron copiadas y estudiadas profusamente y, tras la implantación de la imprenta, fue el autor más editado antes de 1500 (se imprimieron seis veces sus Opera omnia, que recogían sesenta tratados). J.A. Paniagua ha desvelado las claves que explican las causas de su inmenso prestigio, hasta el punto que son apócrifas la mitad de las páginas de sus obras completas editadas durante el Renacimiento.

Nos hallamos ante un autor que abordó una gran variedad de temas, tratándolos todos ellos con singular competencia. Por otro lado, su personalidad responde perfectamente a la convulsionada época en que vivió. Fue el maestro más ilustre de la más prestigiosa escuela de medicina. Sus curaciones sensacionales le ganaron el favor de papas y monarcas, lo que le ofreció una cierta impunidad para publicar sus ideas religiosas, que participaban en los ideales espirituales y de pobreza propugnados por los fraticelos. Arnau fue un hombre de una gran influencia, pero su osadía le valió el ostracismo en los últimos años de su vida y la condena inquisitorial a sus doctrinas religiosas, tras su muerte y la de sus protectores (1316 y 1318). Nada pudieron hacer para evitarlo sus albaceas y seguidores en Valencia, tal y como ha demostrado M. Rodrigo Lizondo.

De lo anteriormente expuesto, no es difícil comprender que Arnau fuese convertido en un personaje legendario, a quien se atribuyó todo tipo de escritos, especialmente alquímicos. Tal «conversión» sufrieron también Ramón Llull y Roger Bacon, figuras coetáneas suyas y de características similares.

La historiografía contemporánea está realizando una rigurosa tarea de expurgo de los numerosos textos falsamente atribuidos al médico valenciano. J.A. Paniagua ha puesto de relieve que de la producción auténticamente original "se desprende una doctrina científica armónica y coherente". Es la expresión del saber impartido en Montpellier, centro de la medicina escolástica basada en la tradición galénica e impulsora de la recuperación del sistema hipocrático-galénico, frente a los seguidores de la sistematización avicenista.

Agustín Rubio y Mateu Rodrigo han destacado la clara influencia de las doctrinas religiosas del médico valenciano entre las capas burguesas ciudadanas, donde alcanzaron gran popularidad las propuestas espirituales de los Beguins, agrupaciones de laicos con ideales espiritualistas, cuya principal actividad era la atención a los pobres en los hospitales. Entre las propuestas de Arnau, una de las más repetidas era la llamada a la caridad hospitalaria y a la construcción de hospitales. En este ambiente, se integran la fundación del Hospital de En Clapers por Bernat de Clapers, uno de los procuradores en la gestión de los negocios de Arnau de Vilanova y hombre vinculado estrechamente al círculo de sus seguidores en la ciudad, en el que también se movió Guillem Ramón Catalá, fundador del Hospital dels Beguins. Hay que destacar que tales fundaciones respondieron asimismo a la convicción de la eficacia de la tarea hospita- 
laria en el proceso de asentamiento de la cultura cristiana entre la mayoría musulmana.

El segundo período en que hemos dividido la actividad científica cristiana en la Valencia bajomedieval, si bien no se interrumpen las relaciones de valencianos con la Universidad de Montpelier, como ya hemos indicado, sí es cierto, que pasan a primer plano las universidades italianas. La principal aportación de las primeras generaciones italianizantes no se dieron en el terreno de la producción escrita, sino en la fundación del colegio de cirugía al que ya hemos aludido. Desde 1433 que existía el gremio de cirujanos de Valencia, pero es en 1462 cuando consigue que el municipio creara una lectura o escola de cirugía y que fuera obligatoria la asistencia para conseguir la licencia municipal correspondiente. La iniciativa partió de médicos universitarios de mentalidad avanzada, algunos de edad madura como el examinador Lluis Dalmau, que fue el primer profesor, el ya mencionado Joan de Bosnia; otros, todavía muy jóvenes, como es el caso de Lluis Alcanyis y Pere Pintor que centraron su actividad en la transición de siglo XV al XVI. El 1478 l'escola, obtuvo la autorización real para disecar cadáveres humanos, hecho de trascendental importancia y que puede considerarse como el punto de partida del posterior florecimiento de la escuela anatómica valenciana. Al integrarse esta institución a la Universidad de Valencia, ejemplo paradigmático de las universidades de tipo municipal, lo que representó la culminación del poder ascendente de la oligarquía urbana en la actividad docente, cultural y científica; esta Universidad se convirtió en una de las primeras de Europa que dispuso de una cátedra de cirugía.

El interés de los reyes de la Corona de Aragón por los estudios universitarios fue grande en personas tales como Jaime II, Alfonso el Benigno, Pedro el Ceremonioso y Martín el Humano. El mismo interés detectamos entre los burgueses que dirigieron la vida ciudadana de Valencia; un patriciado urbano que hizo de la actividad mercantil y del desarrollo del comercio, el eje en torno al que giró la vida social de Valencia, y que estableció relaciones normales con todo el Mediterráneo, desde Beirut y Alejandría a Marsella y Barcelona, y con todo el Atlántico, desde Sevilla y Lisboa a Brujas. Ello le dotó de un evidente cosmopolitismo. A ello se añadió la valoración positiva de la ciencia («molt honren e enoblexen ciutat hòmens ornats de siència») (1401), como pone de relieve el epistolario de los «jurats» de la ciudad de Valencia, recientemente editado (A. Rubio). Si la corona y el patriciado urbano participaron de semejante actitud, ¿por qué siguió manteniéndose el modelo arcaico de la libertad de enseñanza (modelo abierto), en fuerte contraste con la penetración que la ciencia escolástica consiguió en la sociedad valenciana de los siglo XIV y XV, y que nos es conocida a través de las bibliotecas, que se nos han conservado, de médicos y burgueses? Dado el estado actual de las investigaciones, no podemos responder con un mínimo de rigor a estas preguntas. Lo que sí, creo, podemos afirmar es que no existió ciencia en los doscientos cincuenta años que siguieron a la conquista de Valencia por Jaime I, acorde con la situación estratégica de la ciudad (desde el punto de vista geográfico, social y demográfico), y con sus relaciones nacionales e internacionales. Seré más preciso: durante ese período, la sociedad valenciana —algunos de sus miembros-consumió 
ciencia (ciencia hecha fuera de sus territorios), pero no creó ciencia. A pesar de que en la segunda mitad del siglo XV se crearon estructuras propicias para ello. La inexistencia de un Estudio General en Valencia condenó a ésta a lo que con expresión actual llamaríamos provincialismo científico. Sólo salió de esta situación en la transición del siglo XV al XVI, con la creación del Estudi General. Será a partir de esos años cuando detectaremos una actividad científica propiamente dicha.

La actitud de la Iglesia y de las órdenes mendicantes valencianas, posiblemente desempeñaran un papel de cierta relevancia en la situación que hemos descrito.

Ello no quiere decir que la universidad, con su secuela de producción científica, dejara de tener presencia en Valencia. Ahora bien, fue una presencia indirecta. Valencia se ofreció a la universidad escolástica como un mercado donde colocar sus productos; sea en forma de profesionales (teólogos, juristas, médicos, graduados en artes), sea en forma de compradores de libros. Valencia, pues, consumió ciencia, pero no la creó. Los ejemplos más claros los tenemos en las figuras valencianas que participaron en el proceso de creación científico-médica. Nos referimos a los médicos Arnau de Vilanova (c. 1240-1311), Berenguer Eimerich (c. 1290-c. 1356), Nadal Lambrí (m.c. 1395), Joan de Bosnia (fl. 1463-84). Todos ellos estuvieron vinculados a centros universitarios y de ellos recibieron el estímulo intelectual. Arnau de Vilanova y Berenguer Eimerich lo estuvieron a Montpellier, Nadal Lambrí a Lérida, de donde fue catedrático. Antes hemos mencionado el pequeño escrito de Arnau dedicado al obispo de Valencia, Jaspert de Botonac, en lo que parece un reconocimiento a la curiosidad científica del prelado y a una posible actividad de patronazgo; toda su obra restante médica y de filosofía natural, desde sus traducciones de obras médicas islámicas hasta sus comentarios escolásticos y sus escritos clínicos más aplicados, no parece tuvieran nada que ver con el ambiente intelectual o social valencianos, pese al íntimo contacto que mantuvo con la ciudad y con los miembros de su patriciado urbano. Igual podemos decir de Berenguer Eimerich, cuyas obras tienen todas un claro perfil universitario, y de las que nos consta que, una al menos, fue realizada por directa petición de Bernardo de Gordón, su maestro y compañero de claustro de Arnau en Montpellier. Nadal Lambrí, natural de Morella, se formó en Montpellier y Lleida; en esta ciudad ejerció la medicina y de su facultad de medicina fue profesor, participando activamente en las disputas científicas en torno a la doctrina de los tratados medicinales y contribuyendo a ellas con una obra, por desgracia perdida (L. García Ballester, M. R. Mc.Vaugh).

\section{REFERENCIAS BIBLIOGRÁFICAS}

CARDONERY PLANAS, A. (1973), Història de la Medicina a la Corona d'Aragó (11621479), Barcelona, Scientia.

CARRERAS ARTAU, J. (1935), La llibreria d'Arnau de Vilanova. Analecta Sacra Tarraconensis, 11, 63-84.

CARRERAS ARTAU, J. (1950), L'epistolari d'Arnau de Vilanova, Barcelona, Institut d'Estudis Catalans. 
COLIN, G. (1911), La Tedkira d'Abu-l Ala, publiée et traduite..., Paris, Publs. de l'École Supérieure de Lettres d'Alger.

COMENGE, L. (1897), La Medicina en el Reino de Aragón. Siglo XIV. El Siglo Médico, 44, 449-459, 481-484, 497-501, 513-517, 529-533, 545-549, 561-566.

FAURADO DE SAINT GERMAIN, L. (1950), Un lunario valenciano cuatrocentista. Boletín de la Real academia de Buenas Letras de Barcelona, 23, 9-48.

FRANCO SÁNCHEZ, F. y CABELLO, M.S. (1990), Muhammad AS-Safra, el médico y su época, Alicante, Universidad de Alicante.

GALLENT MARCO, M. (1985), El gremi de cirurgians de València: (1310-1499) procés de constitució. Afers, 1, 249-269.

GARCÍA BALLESTER, L. (1982), Arnau de Vilanova (c. 1240-1311) y la reforma de los estudios médicos en Montpellier (1309): el Hipócrates latino y la introducción del nuevo Galeno, Dynamis, 2, 97-158.

GARCÍA BALLESTER, L. (1988), La medicina Valenciana medieval, València, Eds. Alfons El Magnànim, I.V.E.I.

GARCÍA BALLESTER, L;; MC. VAUGH, M.R. y RUBIO, A. (1984), Medical Licensing and Learning in Fourteenth Century Valencia. Transanctions of the American Philosophical Society, 79/6, 1-128.

GARCÍA BALLESTER, L.; PANIAGUA, J.A.; MCVAUCH, M.R. y RUBIO, A. (dirs.) (19751993), Arnaldi de Villanova Opera Medica Omnia, Granada-Barcelona, Universidad de Granada-Universidad de Barcelona.

GARCÍA BALLESTER, L. (1989), La ciencia en Valencia después de la conquista. En: En torno al 750 aniversario. Antecedentes y consecuencias de la conquista de Valencia, València, Consell Valencià de Cultura, Vol. II, pp. 239-254.

LÓPEZ PIÑERO, J.M. y NAVARRO BROTONS, V. (1995), Història de la Ciència al Pais Valencià, València, Edicions Alfons el Magnànim.

MILLÁS VALLICROSA, J.M. (1960), En torno a las tablas astronómicas del rey Pedro IV de Aragón. En: Nuevos estudios sobre la historia de la ciencia española, BarceIona, C.S.I.G., pp. 279-285.

PANIAGUA ARELLANO, J.A. (1969), El maestro Arnau de Vilanova, médico, Valencia, Cátedra e Instituto de Historia de la Medicina.

PANIAGUA ARELLANO, J.A. (1994), Studia Arnaldina. Trabajos en torno a la obra médica de Arnau de Vilanova, c. 1240-1311 Barcelona, Fundación Uriach.

POULLE, E. (1966), Théorie des planètes et trigonommetrie u Xve siècle, d'aprés un équatoire inédite, la sexagenarium. Journal del Savants, 3, 129-161.

PUIG OLIVER, J. de (1980), El procés dels lul.listes valencias contra Nicolau Eimeric en el marc del Cisma d'Occident. Butlletí de la Societat Castellonense de Cultura, $56,319-463$.

ROMANO, D. (1956), Los hermanos Abenmenassé al servicio del Pedro el Grande de Aragón. En: Homenaje a Millás Vallicrosa, Barcelona, C.S.I.C., Vol. II, pp. 243-292. RUBIO VELA, A. (1984), Pobreza, enfermedad y asistencia hospitalaria en la Velencia del siglo XVI. València, Institució Alfons el Magnànim.

SALAVERT FABIANI, V.L. (1988), Los saberes médicos y su enseñanza (Baja Edad 
Media). En: J.M. López Piñero (dir.). Historia de la Medicina Valenciana, Valencia, Vicent García Editores S.A., pp. 71-87.

STEINSCHNEIDER, M. (1883), Aben's-Salt (gest. 1134) und sine Simplicia. Ecin Beitrag Zur Heilmittellehre der Araber. Archiv für pathologischesn Anatomie und Physiologie, 94, 28-65.

VERNET, J. (1989), La ciència abans de la conquesta. En: En torno al 750 aniversario. antecedentes y consecuencias de la conquista de Valencia, València, Consell Valencià de Cultura, Vol. II, pp. 227-239. 